\title{
Effects of Ischemia on Epicardial Segment Shortening ${ }^{1}$
}

\author{
Mack C. Stirling, M.D., Michael Choy, M.D., Thomas B. McClanahan, \\ Robert J. SchotT, M.D., M.S.P.H., and Kim P. Gallagher, Ph.D. \\ Thoracic Surgery Research Laboratory, Departments of Surgery (Thoracic Section) and Physiology, \\ The University of Michigan Medical School, Ann Arbor, Michigan 48109
}

Submitted for publication October 17, 1989

To evaluate the effects of nontransmural ischemia on epicardial contractile function, we implanted sonomicrometers in 15 open-chest, anesthetized (halothane) dogs. One cylindrical crystal (radiating ultrasound $360^{\circ}$ ) was used as a transmitter for three conventional flat plate crystals arrayed to measure epicardial segment shortening along three different axes that were deviated $0^{\circ}$ (parallel), $45^{\circ}$ (oblique), and $90^{\circ}$ (perpendicular) from surface fiber orientation in the anteriorapical or posterior-basal left ventricle. During baseline conditions, epicardial shortening was maximal parallel with fiber orientation. Shortening decreased in a nonlinear manner as deviation from fiber orientation increased, but there were significant differences between the two left ventricular regions suggesting that more substantial lateral strain occurs in the anterior-apical than the posterior-basal area. During coronary inflow restriction, changes in epicardial segment shortening also varied greatly depending on location and alignment. At levels of wall thickening impairment associated with normal subepicardial perfusion, changes in epicardial function were restricted to the segments aligned perpendicular to fiber orientation whereas the parallel and oblique segments displayed moderate dysfunction or none at all. Thus, transmural tethering modifies epicardial segmental motion during coronary inflow restriction, but the severity of the influence depends on the alignment and location of the epicardial measurements. 1991 Academic Press, Inc.

\section{INTRODUCTION}

In several previous studies [1-7], the effects of nontransmural ischemia on epicardial contractile function were examined. The demonstration of epicardial dysfunction when ischemia was limited to deeper myocardial layers by partial coronary stenosis led to the hypothesis that transmural tethering could restrict motion in the normally perfused subepicardial layers of the left

\footnotetext{
${ }^{1}$ Supported in part by the American Heart Association of Michigan and NIH Grant R01 HL32043.
}

ventricular wall. For example, Weintraub et al. [3] reported that epicardial segment lengths showed progressive dysfunction which tended to relate more closely to changes in subendocardial perfusion and contractile function than subepicardial perfusion during coronary stenosis. In other studies, however, the epicardial response to ischemia limited to deep and middle myocardial layers varied more markedly, depending on the orientation of segmental measurements relative to surface fiber orientation $[4,5]$ and location in the left ventricle $[4,6]$. Consequently, the relative importance of transmural tethering on epicardial function during acute ischemia has remained an unsolved issue.

The initial objectives of the present study were (1) to determine how unaxial segment alignment (relative to surface fiber orientation) influenced segment shortening in the epicardium of the left ventricle and (2) to determine whether significant regional variability in this relationship exists by comparing the anterior-apical with the posterior-basal area. After establishing how segmental shortening varied by location and alignment during control conditions, the objective was to evaluate how nontransmural and transmural ischemia effects epicardial segment shortening. We measured epicardial contractile function with sonomicrometers arrayed as segment lengths and produced different levels of ischemia by monitoring changes in transmural wall thickening during partial or complete coronary occlusion. Changes in epicardial segment shortening were used to test the hypothesis that transmural tethering negatively influences systolic function in the subepicardium.

\section{METHODS}

Experimental preparation. The study was performed in 15 open-chest dogs anesthetized with halothane (endtidal concentration $1 \%$ or less) and artificially ventilated. Arterial blood gases and $\mathrm{pH}$ were measured periodically to ensure they were in the normal range and that $\mathrm{PO}_{2}$ exceeded $100 \mathrm{~mm} \mathrm{Hg}$. Intravenous fluids were infused to sustain normal arterial pressures and hematocrits were determined at intervals to make certain hemodilution (hematocrit less than $30 \%$ ) was not pro- 


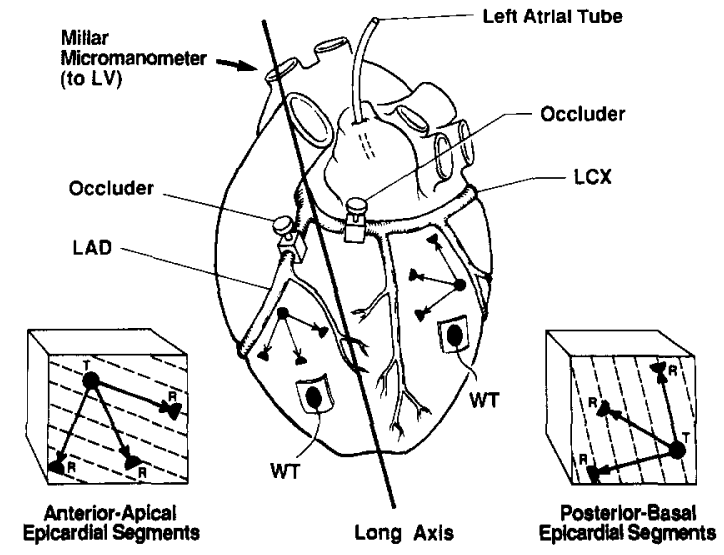

FIG. 1. Diagram of instrumentation used in the open-chest, halothane-anesthetized preparations of this study. The heavy line through the heart indicates the approximate location of the "long axis" of the heart. The insets indicate the location of the ultrasonic crystals in the anterior-apical group (left inset) representing myocardium supplied by left anterior descending (LAD) artery and posterior-basal group (right inset) representing myocardium supplied by the circumflex (LCX) artery. In the epicardium one crystal was used as a transmitter (T) and three crystals acted as receivers ( $R$ ) to simultaneously measure three segment lengths. The axes of the segments were deviated approximately 0,45 , and $90^{\circ}$ from surface fiber orientation, which is represented by the dashed lines. 'Transmural wall thickness (WT) was measured with sonomicrometers, as well, at closely adjacent locations.

duced. The dogs were instrumented as shown in Fig. 1. A Millar high-fidelity micromanometer was passed into the left ventricle via the carotid artery and aorta for measurement of left ventricular pressure. Tygon catheters were placed in the left ventricle (via the apex to verify calibration of the Millar micromanometer in $\mathrm{mm} \mathrm{Hg}$ ) and aorta (via a femoral artery to measure aortic pressure).

In each dog, four sonomicrometers were implanted in the epicardium as shown in Fig. 1 (insets). One cylindrical crystal, radiating ultrasound in $360^{\circ}$ [8] was used as a transmitter for three conventional flat-plate crystals (1.5-2.0 $\mathrm{mm}$ diameter) arrayed to measure segment shortening simultaneously along three axes relative to surface fiber orientation: (1) parallel (i.e., deviated $0^{\circ}$ ), (2) oblique (deviated $45^{\circ}$ ), and (3) perpendicular (deviated $90^{\circ}$ ) to surface fiber orientation. In the remainder of the text, when referring to the three categories of epicardial segments, the terms parallel, oblique, and perpendicular will be used to describe them. The orientations were confirmed at postmortem examination, as previously described, by directly measuring with a protractor the angle between surface fiber direction and the axis through each pair of epicardial crystals after gently stripping away the epicardial sheath of connective tissue $[5,6]$. Additionally, in each dog, a pair of crystals was arrayed to measure wall thickness at a closely adjacent location [6, 9-11]. One crystal of this pair (2-mm diameter) was inserted tangentially through the myocardium to the endocardium; the other crystal (4-mm diameter), attached to a dacron patch, was attached to the epicardium with shallow sutures after locating the position of least distance between the crystals while monitoring the signals with an oscilloscope [10]. We used the thickness mode signal to achieve maximum spatial resolution. The dimension gauges were connected to a Triton (Model 120, Triton Technology, San Diego, CA) sonomicrometer for processing of signals.

The animals were divided into two groups. In eight animals the dimension gauges were positioned in the lateral-posterior wall of the left ventricle at the level of the minor equator or above (designated the posterior-basal group). In seven animals, the gauges were placed in the anterior wall below the level of the minor equator (designated the anterior-apical group). Because ischemia was produced in these experiments, care was taken to locate the sonomicrometers within the areas that would be rendered ischemic by coronary stenosis or occlusion. Each set of sonomicrometers was implanted inside at least one major diagonal or marginal branch of the left anterior descending or circumflex arteries distal to the site of the occluder [6].

Experimental protocol. The same protocol was followed in the posterior-basal and anterior-apical groups. After allowing $30 \mathrm{~min}$ for the animal to stabilize after completing instrumentation, we made control measurements. The control measurements allowed us to evaluate the relationship between fiber orientation and alignment of the sonomicrometers in the posterior-basal and anterior-apical left ventricle. Then we produced progressive levels of partial coronary stenosis while monitoring the wall thickness signal for reductions in systolic wall thickening. Data during coronary stenosis from two dogs in the posterior-basal group and one dog in the anteriorapical group were not used because the wall thickness sonomicrometers were not properly aligned when examined at necropsy. Alterations in wall thickening were used as a guide to produce predictable changes in the distribution of myocardial blood flow across the wall. In addition, wall thickening provided a standard of integrated transmural systolic performance for comparison with epicardial segment shortening [6].

Tracer labeled microspheres were not injected to measure myocardial blood flow. Rather, we relied on the wall thickening measurement as an indirect "flowmeter" of myocardial blood flow distribution [12]. As demonstrated in several previous reports, wall thickening provides a reliable estimate of relative levels of subendocardial perfusion due to the close and direct correlation between these two parameters $[6,12-15]$. Therefore, we think changes in wall thickening can be used reliably as a guide to estimate when subepicardial perfusion is reduced significantly but no attempt is being made to quantify subepicardial blood flow in absolute terms.

Different levels of coronary stenosis were produced in each experiment, the number of levels varying from dog 


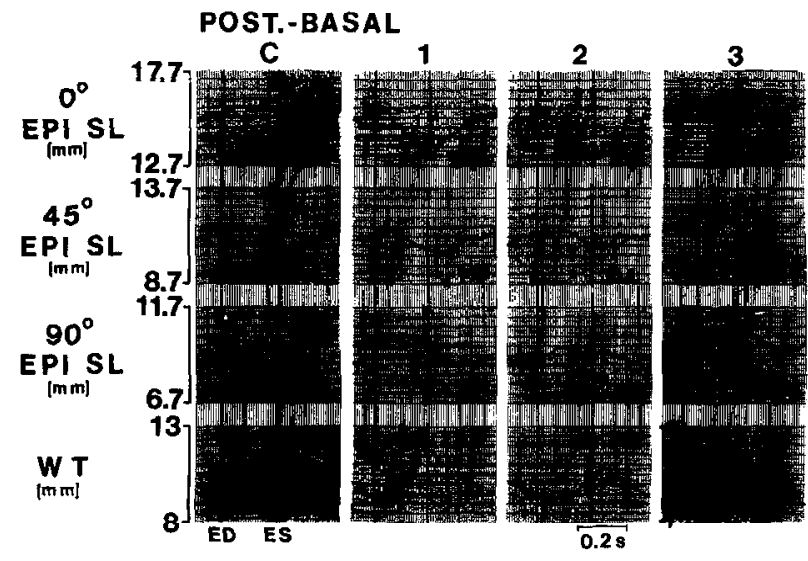

FIG. 2. Example of analog tracings from one experiment in the posterior-basal group (dimension gauges implanted in the basal half of the left ventricle supplied by the left circumflex artery). Epicardial segment lengths (EPI SL) are shown in the upper three rows with average percentage shortening (\% dL) superimposed on the recording. Wall thickness (WT) is presented in the bottom panel. Control (C) or baseline recordings are shown in the first panel. Panels 1,2 , and 3 demonstrate the effects of three levels of coronary inflow restriction corresponding to the three levels of transmural (or wall thickening) dysfunction discussed in the text. The solid vertical lines represent end-diastole (ED) and end-systole (ES).

to dog. We concentrated statistical analysis (in the six dogs from each group with satisfactorily aligned wall thickness sonomicrometers), however, on three specific levels of wall-thickening dysfunction against which transmural blood flow distributions had been correlated in a previous study [6]. Level 1 of regional dysfunction was defined by wall thickening reduced by less than $50 \%$ from control. Level 2 was characterized by further reduction in systolic wall thickening, greater than $50 \%$ from control but not complete elimination of thickening. Finally, a total coronary occlusion was produced, associated with replacement of wall thickening by dyskinesia or paradoxical motion (Level 3 of regional dysfunction).

In the remainder of the text, the terms Level 1 , Level 2 , and Level 3 will be used to refer to the three levels of regional dysfunction which were defined operationally by the changes produced in systolic wall thickening. Levels 1 and 2 were characterized by changes in wall thickening consistent with reduced subendocardial and midmyocardial but normal subepicardial perfusion [6]. Level 3 was characterized by changes in wall thickening consistent with transmural reductions in blood flow [6]. Therefore, alterations in epicardial segment shortening at dysfunction Levels 1 and 2 were used to determine if transmural tethering restricted epicardial motion when subepicardial perfusion was at or near normal levels. The time required for performance of this protocol was approximately $1 \mathrm{hr}$.

At the end of the experiments the dogs were killed with $\mathrm{KCl}$. The heart was removed and placed in formalin for 2 days. Wall thickness dimension gauges were left in the heart to allow careful evaluation of their alignment across the wall. Epicardial dimension gauges, implanted superficially, were removed but small holes $(1-2 \mathrm{~mm}$ in depth) remained in the heart enabling measurement of gauge alignment relative to surface fiber orientation $[5,6]$.

Data analysis. Recordings were made during each experiment on an eight-channel Hewlett-Packard pressurized-ink recorder. Wall thickness, the three epicardial segment lengths, and left ventricular pressure were recorded simultaneously. The variables analyzed were segment length and wall thickness at end-diastole (identified as the point corresponding to the onset of the positive $\mathrm{dP} / \mathrm{dt}$ signal), and end-systole (defined as the point corresponding to maximum excursion at or before 20 msec prior to peak negative $\mathrm{dP} / \mathrm{dt}$ ) [16]. Examples of recordings from experiments in each group are presented in Figs. 2 and 3 to demonstrate the timing of these points. The extent of wall thickening, extent of segment shortening along each of the three axes, peak positive $\mathrm{dP} / \mathrm{dt}$, peak negative $\mathrm{dP} / \mathrm{dt}$, left ventricular systolic and end-diastolic pressures, heart rate, and mean aortic blood pressure were also analyzed.

The extent of segment shortening or wall thickening was calculated as the difference in millimeters between end-diastolic and end-systolic dimensions and was also expressed as a percentage change from the end-diastolic length or wall thickness. End-diastolic epicardial segment lengths during control conditions were normalized to a distance of $10.00 \mathrm{~mm}$ and changes in dimensions were referenced to this value [17]. The reason for this

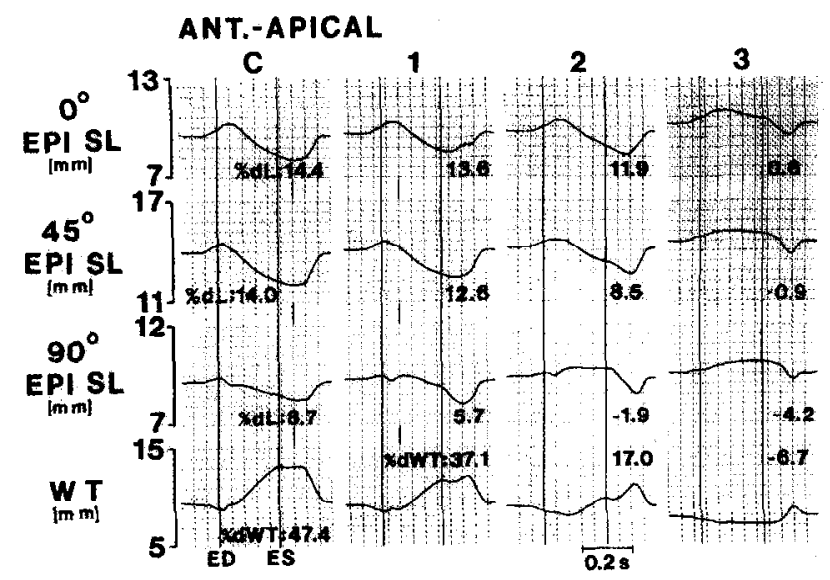

FIG. 3. Example of analog tracings from one experiment in the anterior-apical group (dimension gauges implanted in the apical half of the left ventricle supplied by the left anterior descending artery). Epicardial segment lengths (EPI SL) are shown in the upper three rows with average percentage shortening (\%dL) superimposed on the recording. Wall thickness (WT) and percentage wall thickening (\%dWT) are presented in the bottom panel. Control (C) or baseline recordings are shown in the first panel. Panels 1,2 , and 3 demonstrate the effects of three levels of coronary inflow restriction corresponding to the three levels of transmural dysfunction discussed in the text. The solid vertical lines represent end-diastole (ED) and end-systole (ES). 
normalization was the degree of variability in the distance between epicardial segment length crystals (range $6-18 \mathrm{~mm}$ ), in large part due to the limitations in available implantation sites posed by epicardial vasculature and the location of the wall thickness dimension gauge. Wall thickness dimensions were not normalized.

Data were obtained from high-speed recordings (100 $\mathrm{mm} / \mathrm{sec}$ ) by hand calculation. A minimum of 8 cardiac cycles were averaged at each condition. Hemodynamic and dimension data were analyzed in both anterior-apical and posterior-basal groups at four conditions (Control, Level 1, Level 2, and Level 3) with repeated measures analysis of variance. When overall significance was detected, the Scheffe test was used to discriminate the significance of differences between conditions [18].

Dimension data were also analyzed in both groups at each condition for the three axes (parallel, oblique, and perpendicular) with analysis of variance. When overall significance was detected, paired $t$ tests were used to discriminate the significance of differences between conditions. Since three comparisons were performed, a Bonferroni correction gave $P<0.0167$ as the minimum acceptable alpha level. When " $P<0.05$ " is indicated in tables and text, it represents the corrected value. Comparisons between variables in the anterior and posterior groups were made with unpaired $t$ tests.

Data are reported in the text, table, and figures as means \pm standard deviation.

\section{RESULTS}

Fiber orientation and epicardial segment alignment. The parallel segments were deviated from surface fiber orientation an average of $5 \pm 4^{\circ}$ (range $0-10$ ) in the anterior-apical group and $7 \pm 6^{\circ}$ (range $0-18$ ) in the posterior-basal group. The oblique segments were deviated 42 $\pm 7^{\circ}$ (range 33-53) in the anterior-apical group and 47 $\pm 7^{\circ}$ (range 33-59) in the posterior-basal group. The perpendicular segments were deviated $85 \pm 2^{\circ}$ (range 82 87 ) in the anterior-apical group and $83 \pm 6^{\circ}$ (range 7190 ) in the posterior-basal group. There were no significant differences between the two groups in terms of sonomicrometer alignment relative to fiber orientation.

Epicardial segment shortening during control conditions. Hemodynamic data in the six dogs from each group that underwent progressive stenosis are summarized in Table 1 . The data were nearly the same in the larger number of dogs studied only during baseline conditions. Small but significant differences were evident between the anterior-apical and posterior-basal groups in heart rate, peak systolic pressure, and peak positive $\mathrm{dP} / \mathrm{dt}$. No significant differences were detected in terms of left ventricular end-diastolic pressure, mean arterial blood pressure, and peak negative $\mathrm{dP} / \mathrm{dt}$. The values in both groups were within the normal range for this anesthetized, open-chest preparation [6].
Examples of representative analog tracings from one experiment in the posterior-basal group and one in the anterior-apical group are shown in Figs. 2 and 3, respectively. In addition to analog tracings from the three epicardial segment lengths, wall thickness waveforms are presented in the lower panels of each figure. Percentage shortening or thickening values are superimposed on each panel.

Average segment length data during baseline conditions are summarized in graphical form in Fig. 4. In the posterior-basal group during control conditions, the extent of parallel epicardial segment shortening from enddiastole $(1.09 \pm 0.18 \mathrm{~mm}, P<0.05)$ significantly exceeded oblique shortening $(0.85 \pm 0.05 \mathrm{~mm})$. Perpendicular shortening was more variable but was significantly less than that in the other two alignments, averaging only $0.13 \pm 0.33 \mathrm{~mm}$.

In the anterior-apical group, epicardial segment shortening differed qualitatively and quantitatively from the posterior-basal group. Shortening averaged $1.42 \pm 0.23$ $\mathrm{mm}$ in the parallel segments, $1.33 \pm 0.28 \mathrm{~mm}$ in the oblique segments, and $0.95 \pm 0.34 \mathrm{~mm}$ in the perpendicular segments. There were no significant differences between the parallel and oblique categories or between the oblique and perpendicular categories, but perpendicular shortening was significantly less than shortening in the parallel category (Fig. 4). Anterior-apical epicardial shortening significantly exceeded posterior-basal shortening in all three categories.

Effects of coronary stenosis. Hemodynamic data from the six dogs in each group that underwent progressive coronary stenosis are summarized in Table 1 . No significant changes in heart rate were observed in either the posterior or anterior groups during graded ischemia. Left ventricular peak systolic pressure and mean arterial blood pressure tended to decrease during coronary stenosis but this reached significance only in the posterior group, probably reflecting the larger portion of the left ventricle supplied by the circumflex artery than the left anterior descending artery. Left ventricular end-diastolic pressure increased significantly in both groups at Level 3 (total occlusion) compared with control.

Average data on wall thickness are presented in Table 2. During control conditions, there was a significant difference in end-diastolic and end-systolic wall thickness between the anterior-apical and posterior-basal groups, reflecting the thinner wall that is evident closer to the apex (anterior-apical group). The extent of baseline thickening (dWT) and percentage thickening (\%dWT), however, were not significantly different between anterior-apical and posterior-basal groups. During ischemia, changes produced in wall thickness dimensions paralleled each other closely in both groups (Fig. 2 and 3). The degree of change at every level, evaluated by comparison of raw data or by the extent of relative change, was the same for both groups demonstrating that we had 
TABLE 1

Hemodynamic Data during Baseline Conditions and Three Levels of Regional Dysfunction

\begin{tabular}{|c|c|c|c|c|c|c|}
\hline \multicolumn{7}{|c|}{ Posterior-basal group } \\
\hline HR $(n=6)$ & $130 \pm 8$ & $127 \pm 5$ & NS & $128 \pm 6$ & NS & $129 \pm 7$ \\
\hline LVEDP $(n=6)$ & $8.7 \pm 2.8$ & $9.5 \pm 2.6$ & NS & $10.9 \pm 3.1$ & $<0.05$ & $13.8 \pm 2.4^{*}$ \\
\hline$(+) \mathrm{dP} / \mathrm{dt}(n=4)$ & $1937 \pm 176$ & $1674 \pm 51$ & NS & $1636 \pm 98$ & NS & $1659 \pm 126$ \\
\hline$(-) \mathrm{dP} / \mathrm{dt}(n=4)$ & $2569 \pm 242$ & $2288 \pm 384$ & NS & $1975 \pm 371^{*}$ & NS & $1804 \pm 235^{*}$ \\
\hline HR $(n=6)$ & $103 \pm 14$ & $105 \pm 17$ & NS & $103 \pm 20$ & NS & $107 \pm 21$ \\
\hline $\operatorname{LSVP}(n=6)$ & $106 \pm 6$ & $106 \pm 11$ & NS & $103 \pm 13$ & NS & $99+14$ \\
\hline LVEDP $(n=6)$ & $7.0 \pm 1.4$ & $7.2 \pm \quad 1.5$ & NS & $7.9 \pm 1.9$ & $<0.05$ & $11.0 \pm 3.0^{*}$ \\
\hline$(+) \mathrm{dP} / \mathrm{dt}(n=3)$ & $1654 \pm 352$ & $1665 \pm 276$ & NS & $1579 \pm 270$ & NS & $1693 \pm 235$ \\
\hline$(-) \mathrm{dP} / \mathrm{dt}(n=3)$ & $2078 \pm 177$ & $2162 \pm 339$ & NS & $2021 \pm 374$ & NS & $1956 \pm 318$ \\
\hline
\end{tabular}

Note. HR, heart rate (heats/min); I.VSP, left ventricular systolic pressure ( $\mathrm{mm} \mathrm{Hg}$ ); I.VFDP, left ventricular end-diastolic pressure (mm $\mathrm{Hg}) ;(+) \mathrm{dP} / \mathrm{dt}$, peak positive first derivative of left ventricular pressure $(\mathrm{mm} \mathrm{Hg} / \mathrm{s}) ;(-) \mathrm{dP} / \mathrm{dt}$, peak negative first derivative of left ventricular pressure ( $\mathrm{mm} \mathrm{Hg} / \mathrm{sec}$ ); $P$, probability of difference between groups (Scheffe test); , significantly different from baseline value (Scheffe test); NS, nonsignificant; data presented as means \pm SD.

achieved our objective of producing comparable levels of transmural dysfunction in the posterior and anterior groups. The relative reductions from baseline values in percentage systolic wall thickening were $35 \pm 6,76 \pm 6$, and $112 \pm 8 \%$ (greater than $100 \%$ indicating dyskinesia or net systolic thinning) at Levels 1,2 , and 3 , respectively, in the posterior-basal group and $31 \pm 9,78 \pm 7$, and $121 \pm 14 \%$ in the anterior-apical group.

Average data on epicardial segment lengths in the three orientations during coronary inflow restriction are summarized in Table 3 (posterior-basal group) and Table 4 (anterior-apical group). The absolute and relative

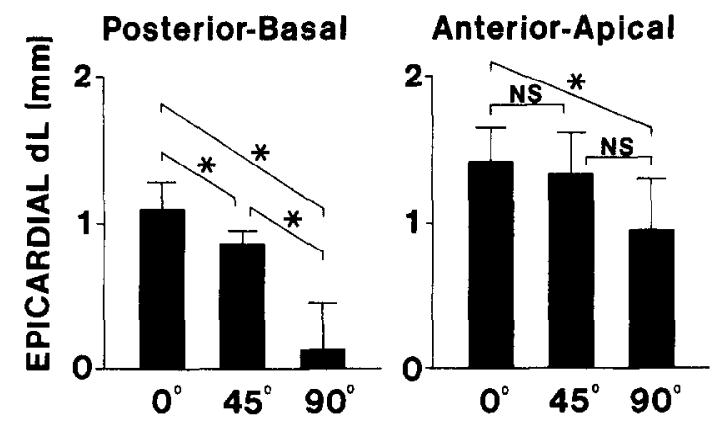

FIG. 4. Average ( \pm SD) epicardial shortening (dL) data in the three categories defined by deviation from surface fiber orientation. Posterior-basal data are shown on the left; anterior-apical data are shown on the right. The distribution of the posterior-basal group data suggests that shortening was closely related to fiber direction. Because perpendicular $\left(90^{\circ}\right)$ shortening was negligible on the average, we think lateral strain was minimal in this group. In the anterior-apical group, parallel $\left(0^{\circ}\right)$ and oblique $\left(45^{\circ}\right)$ shortening were not significantly different and considerable perpendicular shortening was evident suggesting that shortening is less closely related to fiber direction and that a substantial component of shortening occurs in the anteriorapical epicardium lateral to the direction of the surface fibers. changes in epicardial shortening are plotted in Fig. 5 versus relative changes in percentage wall thickening.

In the posterior-basal group, each of the three epicardial segment orientations exhibited different responses to coronary inflow restriction. Similar to our previous observations $[5,6]$ parallel shortening was not reduced at dysfunction Levels 1 and 2 (Table 3), averaging 109 \pm 8 and $116 \pm 17 \%$ of baseline values (Fig. 5), respectively. At the third level of dysfunction, when wall thickening was impaired enough to indicate that subepicardial perfusion was reduced, parallel epicardial shortening decreased to $8 \pm 21 \%$ of the baseline value. In the oblique $\left(45^{\circ}\right)$ alignment, shortening was not significantly changed at the first level of dysfunction (Table 3) but was significantly reduced to $41 \pm 22 \%$ of baseline values at the second level of dysfunction (nontransmural ischemia). At Level 3 (transmural ischemia), oblique shortening was replaced by thinning (Fig. 5). The perpendicular segments in the posterior-basal group shortened negligibly during baseline conditions (Fig. 4, Table 3). The limited systolic shortening evident during baseline conditions was eliminated at dysfunction Level 1 (nontransmural ischemia) and replaced by lengthening in a pattern that contrasts with epicardial shortening in both the 0 and $45^{\circ}$ alignments (Fig. 5).

The anterior-apical segment length data during coronary inflow restriction are summarized in Table 4. Rather than exhibiting relatively distinct patterns like their counterparts in the posterior-basal group, the parallel and oblique anterior-apical segments responded to inflow restriction in a similar manner (Fig. 5).

Parallel shortening was reduced by $19 \pm 9(P$ NS) and $40 \pm 14 \%(P<0.01)$ from baseline at dysfunction Levels 1 and 2 (nontransmural ischemia). These reductions were significantly different $(P<0.01$, unpaired $t$-test 
TABLE 2

Wall Thickness Data during Baseline Conditions and Three Levels of Regional Dysfunction

\begin{tabular}{|c|c|c|c|c|c|c|}
\hline \multicolumn{7}{|c|}{ Basal-posterior group $(n=6)$} \\
\hline EDW & $10.68 \pm 1.86$ & $10.46 \pm 1.89$ & NS & $10.07 \pm 1.84^{*}$ & $<0.05$ & $9.38 \pm 1.98^{*}$ \\
\hline $\mathrm{dWT}$ & $2.50 \pm 0.53$ & $1.58 \pm 0.37^{*}$ & $<0.05$ & $0.57 \pm 0.20^{*}$ & $<0.05$ & $-0.26 \pm 0.19^{*}$ \\
\hline$\% \mathrm{dW} T$ & $23.8 \pm 6.2$ & $15.5 \pm 4.3^{*}$ & $<0.05$ & $5.6 \pm 1.8^{*}$ & $<0.05$ & $-2.9 \pm 2.3^{*}$ \\
\hline $\mathrm{dWT}$ & $2.69 \pm 1.01$ & $1.81 \pm 0.74$ & $<0.05$ & $0.55 \pm 0.27^{*}$ & $<0.05$ & $-0.40 \pm 0.20^{*}$ \\
\hline$\% \mathrm{dWT}$ & $32.1 \pm 10.8$ & $21.9 \pm 8.1^{*}$ & $<0.05$ & $7.0 \pm 3.4^{*}$ & $<0.05$ & $-5.7 \pm 3.1^{*}$ \\
\hline
\end{tabular}

Note. EDW, end-diastolic wall thickness (mm); ESW, end-systolic wall thickness (mm); dWT, ESW-EDW (mm); \%dWT, (dWT/EDW) $\times 100 ; P$, probability of difference between groups (Scheffe test): ${ }^{*}$, significantly different from baseline value (Scheffe test); NS, nonsignificant; data presented as means $\pm \mathrm{SD}$.

comparison) from the relative change in posterior-basal parallel shortening. With transmural ischemia (dysfunction Level 3), dyskinesia was observed in the anterior-apical parallel segments. The changes in the anterior-apical oblique segments were not significantly different from the changes in parallel shortening. Oblique shortening decreased from baseline by $13 \pm 11$ ( $P$ NS) and $41 \pm 21 \%(P<0.01)$ at dysfunction Levels 1 and 2 (nontransmural ischemia). With transmural ischemia (dysfunction Level 3) oblique shortening was replaced by lengthening ( $131 \pm 17 \%$ reduction from baseline).

The perpendicular segments in the anterior-apical group were characterized by significantly greater shortening at baseline than the counterpart segments in the posterior-basal area (Fig. 4). Shortening from end-diastole decreased from baseline by $46 \pm 42,117 \pm 68$, and $170 \pm 35 \%$ at dysfunction Levels 1,2 , and 3 . Perpendicular shortening in the anterior-apical group was more sensitive to ischemia than the parallel and oblique anterior-apical alignments, because shortening was impaired significantly more at dysfunction Levels 1 and 2 (Fig. 5).

\section{DISCUSSION}

Epicardial contractile function has been measured with a variety of techniques $[1-7,19-33]$. In the present study, we implanted sonomicrometers in three different alignments relative to surface fiber direction to deter-

TABLE 3

Epicardial Segment Length Data from the Posterior-Basal Area $(\boldsymbol{n}=6)$ during Baseline Conditions and Three Levels of Regional Dysfunction

\begin{tabular}{|c|c|c|c|c|c|c|}
\hline & Baseline & Level 1 & $P$ & Level 2 & $P$ & Level 3 \\
\hline \multicolumn{7}{|c|}{$0^{\circ}$ (parallel) segment length } \\
\hline EDL & 10.00 & $10.05 \pm 0.06$ & NS & $10.20 \pm 0.13^{*}$ & $<0.05$ & $10.42 \pm 0.20^{*}$ \\
\hline $\mathrm{dL}$ & $1.04 \pm 0.17$ & $1.13 \pm 0.17$ & NS & $1.22+0.36$ & $<0.05$ & $0.08 \pm 0.20^{*}$ \\
\hline$\% \mathrm{dL}$ & $10.4 \pm 1.7$ & $11.2 \pm 1.7$ & NS & $12.0 \pm 3.6$ & $<0.05$ & $0.8 \pm 1.9^{*}$ \\
\hline \multicolumn{7}{|c|}{$45^{\circ}$ (oblique) segment length } \\
\hline$\% \mathrm{dL}$ & $8.8 \pm 0.7$ & $7.6 \pm 1.4$ & $<0.05$ & $3.5 \pm 1.8^{*}$ & $<0.05$ & $-4.5 \pm 2.8^{*}$ \\
\hline \multicolumn{7}{|c|}{$90^{\circ}$ (perpendicular segment length) } \\
\hline EDL & 10.00 & $10.06 \pm 0.12$ & $<0.05$ & 10.39 上 $0.28^{*}$ & NS & $10.57 \pm 0.07^{*}$ \\
\hline ESL & $9.89 \pm 0.37$ & $10.20 \pm 0.37$ & $<0.05$ & $11.04 \pm 0.52^{*}$ & NS & $11.27 \pm 0.23^{*}$ \\
\hline $\mathrm{dL}$ & $0.12 \pm 0.37$ & $-0.14 \pm 0.38$ & $<0.05$ & $-0.65 \pm 0.39^{*}$ & NS & $-0.70 \pm 0.24^{*}$ \\
\hline \%dL & $1.2 \pm 3.7$ & $-1.4 \pm 3.8$ & $<0.05$ & $-6.2 \pm 3.8^{*}$ & NS & $-7.4 \pm 2.8^{*}$ \\
\hline
\end{tabular}

Note. FDL, end-diastalic length (mm); ESL, end-systolic length (mm); dL, EDL-ESL (mm); \%dL, (dL/EDL) $\times 100 ; P$, probability of difference between groups (Scheffe test); ${ }^{*}$, significantly different from baseline values (Scheffe test); NS, nonsignificant; data presented as means $\pm \mathrm{SD}$. 
TABLE 4

Epicardial Segment Length Data from the Anterior-Apical Area $(n=6)$ during Baseline Conditions and Three Levels of Regional Dysfunction

\begin{tabular}{|c|c|c|c|c|c|c|}
\hline & Baseline & Level 1 & $P$ & Level 2 & $P$ & Level 3 \\
\hline \multicolumn{7}{|c|}{$0^{\circ}$ (parallel) segment length } \\
\hline EDL & 10.00 & $10.06 \pm 0.07$ & NS & $10.16 \pm 0.11^{*}$ & $<0.05$ & $10.68 \pm 0.15^{*}$ \\
\hline $\mathrm{dL}$ & $1.44 \pm 0.25$ & $1.16 \pm 0.26$ & NS & $0.87 \pm 0.29^{*}$ & $<0.05$ & $-0.41 \pm 0.39^{*}$ \\
\hline$\% \mathrm{dL}$ & $14.4 \pm 2.5$ & $11.5 \pm 2.6$ & NS & $8.5 \pm 2.8^{*}$ & $<0.05$ & $-3.8 \pm 3.7^{*}$ \\
\hline \multicolumn{7}{|c|}{$45^{\circ}$ (oblique) segment length } \\
\hline dL & $1.32 \pm 0.31$ & $1.14 \pm 0.26$ & NS & $0.73 \pm 0.19^{*}$ & $<0.05$ & $-0.40 \pm 0.22^{*}$ \\
\hline$\% \mathrm{dL}$ & $13.2 \pm 3.1$ & $11.4 \pm 2.6$ & $<0.05$ & $7.2 \pm 1.8^{*}$ & $<0.05$ & $-3.8 \pm 2.1^{*}$ \\
\hline \multicolumn{7}{|c|}{$90^{\circ}$ (perpendicular) segment length } \\
\hline EDL & 10.00 & $10.06 \pm 0.10$ & NS & $10.26 \pm 0.16^{*}$ & $<0.05$ & $10.57 \pm 0.23^{*}$ \\
\hline ESL & $9.17 \pm 0.18$ & $9.61 \pm 0.37$ & $<0.05$ & $10.44 \pm 0.46^{*}$ & $<0.05$ & $11.12 \pm 0.31^{*}$ \\
\hline $\mathrm{dL}$ & $0.83 \pm 0.17$ & $0.46 \pm 0.36$ & $<0.05$ & $-0.18 \pm 0.46^{*}$ & NS & $-0.55 \pm 0.24^{*}$ \\
\hline$\% \mathrm{dL}$ & $8.3 \pm 1.7$ & $4.5 \pm 3.5$ & $<0.05$ & $-1.8 \pm 4.5^{*}$ & NS & $-5.3 \pm 2.4^{*}$ \\
\hline
\end{tabular}

Note. EDL, end-diastolic length $(\mathrm{mm})$; ESL, end-systolic length $(\mathrm{mm}) ; \mathrm{dL}, \mathrm{EDL}-\mathrm{ESL}(\mathrm{mm}) ; \% \mathrm{dL}$, (dL/EDL) $\times 100 ; P$, probability of difference between groups (Scheffe test); ${ }^{*}$, significantly different from baseline values (Scheffe test); NS, nonsignificant; data presented as Ineans $\pm \mathrm{SD}$.

mine the affect of gauge alignment and location on the extent of systolic shortening during normal and ischemic conditions. By restricting our consideration of this issue to the epicardium, the problems associated with verifying fiber orientation at different depths through the wall were minimized [34]. The main objective of this study was to determine how acute ischemia effects epicardial segment shortening in different alignments relative to surface fiber orientation. In so doing, we tested the hypothesis that transmural tethering influences epicardial motion when coronary inflow is restricted.

Baseline epicardial function. During baseline conditions, the effect of gauge alignment relative to fiber orientation on epicardial systolic shortening was different in the posterior-basal and anterior-apical locations (Fig. 4). A factor contributing to the regional differences is the amount of "lateral strain" that appears to occur during baseline conditions. Freeman et al. [35] defined lateral strain as motion directed sideways to fiber orientation in a report on the shortening-fiber orientation relationship in the midwall layers of the left ventricle. Deviations from the relation predicted by Freeman et al. [35], for example, imply that motion directed perpendicular to fiber orientation is occurring. If the data approximate the predicted sigmoidal relationship between shortening and fiber direction, they indicate that shortening is largely in the direction of the fibers. Matre et al. [36] reported comparable results based on studies of segment shortening in anesthetized cats, providing additional support for the validity of the conclusions of Freeman et al. [35].

The distribution of the posterior-basal data (Fig. 4) support the contention that epicardial segment shorten- ing in this area is predominantly parallel with fiber orientation. There appeared to be relatively little lateral strain in the posterior-basal area because perpendicular shortening, a direct measurement that corresponds to lateral strain, was negligible (Fig. 4). Anterior epicardial shortening, on the other hand, appeared less closely related to fiber direction and more strongly affected by lateral strain than the posterior-basal segments. Particularly striking was the amount of shortening measured perpendicular to the surface fibers in the $90^{\circ}$ category (Fig. 4).

We speculate that the differences in perpendicular segment shortening, or lateral strain, reflect differences in shear angle between segment measurements in the two areas. Arts et al. [19], using magnetic field generating coils, measured epicardial ejection phase shortening and shear angle in the anterior left ventricular wall of open-chest dogs. They documented that there was a shear angle of approximately 0.2 radian $\left(11.5^{\circ}\right)$ that could be attributed, at least in part, to torsion or twisting of the left ventricular apex relative to the base [19, 37]. Because $90^{\circ}$ shortening was greater in the anterior-apical region than the posterior-basal area, our results suggest that shear angle change and the amount of torsion are larger in the anterior-apical and than the posterior region. Lacking direct measurements of shear angle, however, we cannot state these conclusions with certainty.

Ischemic epicardial function. Transmural tethering can be defined as restriction of epicardial motion despite normal or near-normal subepicardial perfusion. Therefore, epicardial segment shortening at dysfunction Levels 1 and 2 was the crucial measurement in the present 


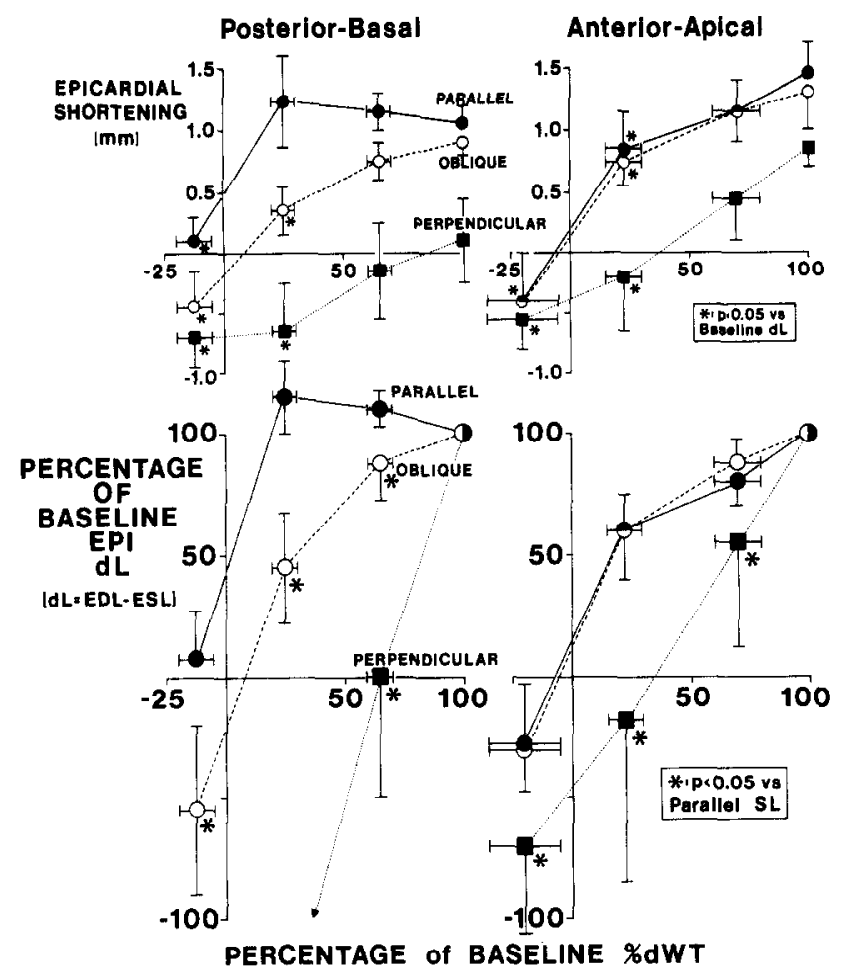

FIG. 5. Relationships between absolute epicardial segment shortening (dL, in millimeters) and changes in percentage wall thickening (\%dWT) produced by progressive coronary stenosis (upper graphs) and between relative changes in epicardial shortening and changes in wall thickening (lower graphs). Data from the posterior-basal group are shown on the left; data from the anterior-apical group are shown on the right. In the posterior-basal group, three distinctive patterns of change characterized the epicardial response to ischemia in the three categories of segments (defined by alignment relative to fiber direction). In the anterior-apical group, the parallel and oblique segments displayed similar patterns. The perpendicular segments in both groups appeared more sensitive to myocardial ischemia than the other segment categories.

study to pursue this objective. Although we did not measure myocardial blood flow directly with microspheres in the present study, ample precedent exists in the literature documenting the utility of changes in percentage wall thickening as an index of transmural perfusion [6, 12-15]. Dysfunction Levels 1 and 2 were characterized by wall thickening changes consistent with subendocardial and midmyocardial, but not subepicardial ischemia.

The posterior-basal epicardial segments displayed three distinctive patterns of change with progressive coronary narrowing. The anterior-apical segments displayed patterns that differed from one another less dramatically (Fig. 5). We observed a tendency for reduced shortening at dysfunction Level 1 and significantly reduced shortening at dysfunction Level 2 in all but the posterior-basal parallel segments (Tables 3 and 4). The present results are consistent with our previous findings that were restricted to evaluation of parallel shortening in the apical and basal areas [6] and shortening deviated approximately $50-70^{\circ}$ from surface fiber orientation in the basal region [5].

Because epicardial shortening decreased significantly in five of the six categories when subepicardial perfusion would have been normal at dysfunction Level 2 (Fig. 5), we conclude that transmural tethering does change epicardial motion. Because the changes in epicardial shortening were not significant at dysfunction Level 1, our data also suggest that the effects of transmural tethering are not striking. In addition, the degree to which nonischemic epicardial dysfunction varied was dramatic. At dysfunction Level 2 we observed epicardial motion that ranged from normal (posterior-basal parallel segments) to severe dyskinesia (perpendicular segments) depending on gauge alignment and location in the left ventricle. Although the relative changes in perpendicular segment shortening were large at dysfunction Level 2, the parallel and oblique segments tended to underestimate the degree of transmural dysfunction (Fig. 5). Consequently, the apparent severity of transmural tethering will depend strongly on how and where segmental epicardial function is measured.

A practical question of some importance is whether or not transmural tethering amplifies the extent of transmural dysfunction. Although we have concluded that transmural tethering does modify epicardial motion, we also interpret our data to suggest that the change in epicardial motion does not add much to the level of transmural dysfunction produced by subendocardial and midmyocardial ischemia alone. Dysfunction Level 2, for example, was characterized by wall thickening reductions that averaged approximately $75 \%$ in both groups. Nonetheless, the pattern of epicardial motion differed significantly in the two groups (Fig. 5). Because two different patterns of epicardial motion were associated with the same degree of wall thickening change, the data suggest that what happens in the epicardium may not be very important. We propose that tethering-related modification of epicardial motion contributes little to the overall functional deficit because the outer wall contribution to total wall thickening is small even during baseline conditions [11, 33, 38-40]. Additional investigation will be required, using outer wall-thickening or multidimensional techniques $[41,42]$, to test this possibility more rigorously.

The nature of transmural tethering remains obscure. Although elegant studies have demonstrated the presence of extensive lateral connections between myocardial cells [43], their physiologic significance is not well defined. Therefore, providing a satisfactory explanation for different epicardial responses (or for the same responses in different locations) is not currently possible. Factors potentially contributing to differences between basal and apical epicardial locations have been discussed in earlier papers $[4,6]$. They include differences in terms of wall thickness, radius of curvature, fiber orientation, and proximity to the rigid valvular rings. 
In conclusion, the results of this experiment show differences between the anterior-apical and posterior-basal regions of the left ventricle, during baseline conditions and during coronary inflow restriction. With nontransmural ischemia, changes in epicardial segment shortening varied greatly depending on their location in the left ventricle and alignment relative to surface fiber orientation. Severe dysfunction was observed only in the perpendicular segments, whereas the parallel and oblique segments displayed responses ranging from no change to moderate reductions in shortening. Therefore, we conclude that transmural tethering does modify subepicardial segmental motion during nontransmural ischemia but the apparent severity of the influence depends on the alignment and location of the epicardial measurements. Additional investigation will be required to establish whether or not the variable effects of tethering have significant consequences in terms of transmural function.

\section{ACKNOWLEDGMENTS}

We express our appreciation to Russell A. Grinage for expert technical assistance and Tarry Goble and Jennifer Edwards for word processing of the manuscript.

\section{REFERENCES}

1. Downey, J. M. Myocardial contractile force as a function of coronary blood flow. Amer. J. Physiol. 230: 1, 1976.

2. Forman, R., Kirk, E. S., Downey, J. M., and Sonnenblick, E. H. Nitroglycerin and heterogeneity of myocardial blood flow. Reduced subendocardial blood flow and ventricular contractile force. J. Clin. Invest. 52: 905, 1973.

3. Weintraub, W. S., Hattori, S., Agarwal, J. B., Bodenheimer, M. M., Banka, V. S., and Helfant, R. H. The relationship between myocardial blood flow and contraction by myocardial layer in the canine left ventricle during ischemia. Circ. Res. 48: $430,1981$.

4. Hattori, S., Weintraub, W. S., Agarwal, J. B., Bodenheimer, M. M., Ranka, V. S., and Helfant, R. H. Contrasting ischemic contraction patterns by zone and layer in canine myocardium. Amer. J. Physiol. 243 (Heart Circ. Physiol. 12): H852, 1982.

5. Gallagher, K. P., Osakada, G., Hess, O. M., Koziol, J. A., Kemper, W. S., and Ross, J., Jr. Subepicardial segment function during coronary stenosis and the role of myocardial fiber orientation. Circ. Res. 50: 352, 1982.

6. Gallagher, K. P., Stirling, M. C., Choy, M., Szpunar, C. A., Gerren, R. A., Botham, M. J., and Lemmer, J. H. Dissociation between epicardial and transmural function during acute myocardial ischemia. Circulation 71: 1279, 1985.

7. Prinzen, R. W., Arts, T., Van der Vusse, G. J., Coumans, W. A., and Reneman, R. S. Gradients in fiber shortening and metabolism across ischemic left ventricular wall. Amer. J. Physiol. 250 (Heart Circ. Physiol. 19): H255, 1986.

8. Heimisch, W., Hagl, S., Gebhardt, K., Meisner, H., Mendler, N., and Sebening, F. Direct measurement of cyclic changes in regional wall geometry in the left ventricle of the dog. Innov. Tech. Biol. Med. 2: 487, 1981.

9. Bugge-Asperheim, B., Leraand, S., and Kiil, F. Local dimension changes of the myocardium measured by ultrasonic technique. Scan. J. Clin. Lab. Invest. 24: 361, 1969.

10. Sasayama, S., Franklin, D., Ross, J., Jr., Kemper, W. S., and McKown, D. Dynamic changes in left ventricular wall thickness and their use in analyzing cardiac function in the conscious dog. Amer. J. Cardiol. 38: 870, 1976.

11. Gallagher, K. P., Osakada, G., Matsuzaki, M., Miller, M. M., Kemper, W. S., and Ross, J., Jr. Nonuniformity of inner and outer systolic wall thickening. Amer. J. Physiol. 249 (Heart Circ. Physiol. 18): H241, 1985.

12. Canty, J. M., Jr. Coronary pressure-function and steady-state pressure-flow relations during autoregulation in the unanesthetized dog. Circ. Res. 63: 821, 1988.

13. Gallagher, K. P., Kumada, T., Koziol, J. A., McKown, M., Kemper, W. S., and Ross, J., Jr. Significance of regional wall thickening abnormalities relative to transmural myocardial perfusion. Circulation 62: 1266, 1980.

14. Gallagher, K. P., Matsuzaki, M., Koziol, J. A., Kemper, W. S., and Ross, J., Jr. Regional myocardial perfusion and wall thickening during ischemia in conscious dogs. Amer. J. Physiol. 247 (Heart Circ. Physiol. 16): H727, 1984.

15. Gallagher, K. P., Matsuzaki, M., Osakada, G., Kemper, W. S., and Ross, J., Jr. Effect of exercise on the relationship between myocardial blood flow and systolic wall thickening in dogs with acute coronary stenosis. Circ. Res. 52: 716, 1983.

16. Gallagher, K. P., Kumada, T., Battler, A., Kemper, W. S., and Ross, J., Jr. Isoproterenol induced myocardial dysfunction in dogs with coronary stenosis. Amer. J. Physiol. 242 (Heart Circ. Physiol. 11): H260, 1982.

17. Theroux, P., Franklin, D., Ross, J., Jr., and Kemper, W. S. Regional myocardial function during acute coronary occlusion and its modification by pharmacologic agents in the dog. Circ. Res. 35: 896, 1974.

18. Glantz, S. A. Primer of Biostatistics. New York: McGraw-Hill, 1987. Pp. 88-91.

19. Arts, 'T., Veenstra, P. C., and Reneman, R. S. Epicardial deformation and left ventricular wall mechanics during ejection in the dog. Amer. J. Physiol. 243 (Heart Circ. Physiol. 12): H379, 1982.

20. Banka, V. S., Bodenheimer, M. M., and Helfant, R. H. Relation between progressive decreases in regional coronary perfusion and contraction abnormalities. Amer. J. Cardiol. 40: 200, 1977.

21. Brower, R. W., Ten Katen, H. J., and Meester, G. T. Direct method for determining regional myocardial shortening after bypass surgery from radiopaque markers in man. Amer. J. Cardiol. 41: $1222,1978$.

22. Harrison, D. C., Goldblatt, A., and Braunwald, E. Studies on cardiac dimensions in intact, unanesthetized man. I. Description of techniques and their validation. Circ. Res. 13: 448, 1963.

23. Heikkila, J., Tabakin, B. S., and Hugenholtz, P. G. Quantification of function in normal and infarcted regions of the left ventricle. Cardiovasc. Res. 6: 516, 1972.

24. Ingels, N. B., Jr., Daughters, G. T., II, Davies, S. R., and MacDonald, I. B. Stereo photogrammetric studies on the dynamic geometry of the canine left ventricular epicardium. J. Biomech. 4: $541,1971$.

25. McDonald, I. G. The shape and movements of the human left ventricle during systole. A study by cineangiography and by cineradiography of epicardial markers. Amer. J. Cardiol. 26: 221, 1970.

26. Nellis, S. H., and Licdtke, A. J. Development and use of a new high-frequency, low mechanical impedance strain gauge. Amer. J. Physiol. 236 (Heart Circ. Physiol. 5): H657, 1979.

27. Wyatt, H. L., Forrester, J. S., Tyberg, J. V., Goldner, S., Logan, S. E., Parmley, W. W., and Swan, H. J. C. Effect of graded reduc- 
tions in regional coronary perfusion on regional and total cardiac function. Amer. J. Cardiol. 36: 185, 1975.

28. Banka, V. S., Yamazaki, H., Agarwal, J. B., Bodenheimer, M. M., and Helfant, R. H. Effects of digitalis on subendocardial and subepicardial dysfunction during acute ischemia. Circulation 65: 1315, 1982.

29. Bertha, B. G., and Folts, J. D. Subendocardial and subepicardial segmental function changes in the dog heart due to gradual coronary flow reduction by an acutely developing thrombus. Cardiovasc. Res. 19: 495, 1985 .

30. Crozatier, B. Relations between myocardial blood flow and postextrasystolic potentiation in epicardial and endocardial left ventricular regional function early after coronary occlusion in dogs. Circulation 66: 938, 1982.

31. LeWinter, M. M., Kent, R. S., Kroener, J. M., Carew, T. C., and Covell, J. W. Regional differences in myocardial performance in the left ventricle of the dog. Circ. Res. 37: 191, 1975.

32. Kerber, R. E., Martins, J. B., Gascho, J. A., Marcus, M. L., and Grayzel, J. Effect of direct-current countershocks on regional myocardial contractility and perfusion. Experimental studies. Circulation 63: 323, 1981.

33. Sabbah, H. N., Marzilli, M., and Stein, P. D. The relative role of subendocardium and subepicardium in left ventricular mechanics. Amer. J. Physiol. (Heart Circ. Physiol. 9): H920, 1981.

34. Streeter, D. D., Spotnitz, H. M., Patel, D. P., Ross, J., Jr., and Sonnenblick, E. H. Fiber orientation in the canine left ventricle during diastole and systole. Circ. Res. 24: 339, 1969.

35. Freeman, G. L., LeWinter, M. M., Engler, R. L., and Covell,
J. W. Relationship between myocardial fiber direction and segment shortening in the midwall of the canine left ventricle. Circ. Res. 56: 31, 1985.

36. Matre, K., Hexeberg, E., and Lekven, J. Fibre orientation in the left ventricle and its influence on local pressure-length loop analysis in cats. Clin. Physiol. 6: 293, 1986.

37. Arts, T., Meerbaum, S., Reneman, R. S., and Corday, E. Torsion of the left ventricle during the ejection phase in the intact dog. Cardiovasc. Res. 18: 183, 1984.

38. Myers, J. H., Stirling, M. C., Choy, M., Buda, A. J., and Gallagher, K. P. Direct measurement of inner and outer wall thickening dynamics with epicardial echocardiography. Circulation 74: 164, 1972.

39. Bolli, R., Patel, B. S., Hartley, C. J., Thornby, J. I., Jeroudi, M. O., and Roberts, R. Nonuniform transmural recovery of contractile function in stunned myocardium. Amer. J. Physiol. 257 (Heart Circ. Physiol. 26): H375, 1989.

40. Homans, D. C., Sublett, E., Lindstrom, P., Nesbitt, T., and Bache, R. J. Subendocardial and subepicardial wall thickening during ischemia in exercising dogs. Circulation 78: 1267, 1988.

41. Villareal, F. J., Waldman, L. K., and Lew, W. Y. W. Technique for measuring regional two-dimensional finite strains in canine left ventricle. Circ. Res. 62: 711, 1988.

42. Waldman, L. K., Fung, Y. C., and Covell, J. W. Transmural myocardial deformation in the canine left ventricle. Normal in-vivo three-dimensional finite strains. Circ. Res. 57: 152, 1985.

43. Borg, T. K., and Caulfield, J. B. The collagen matrix of the heart. Fed. Proc. 40: 2037, 1981. 